\title{
Reflets
}

Revue ontaroise d'intervention sociale et communautaire

\section{Intervenir en contexte(s) minoritaire(s)}

\section{Michèle Kérisit et Gertrude Mianda}

Volume 4, numéro 1, printemps 1998

Intervention en contextes minoritaires

URI : https://id.erudit.org/iderudit/026194ar

DOI : https://doi.org/10.7202/026194ar

Aller au sommaire du numéro

Éditeur(s)

Reflets : Revue ontaroise d'intervention sociale et communautaire

ISSN

1203-4576 (imprimé)

1712-8498 (numérique)

Découvrir la revue

Citer ce document

Kérisit, M. \& Mianda, G. (1998). Intervenir en contexte(s) minoritaire(s). Reflets, 4(1), 10-18. https://doi.org/10.7202/026194ar

Tous droits réservés (C) Reflets : Revue ontaroise d'intervention sociale et communautaire, 1998
Ce document est protégé par la loi sur le droit d'auteur. L'utilisation des services d'Érudit (y compris la reproduction) est assujettie à sa politique d'utilisation que vous pouvez consulter en ligne.

https://apropos.erudit.org/fr/usagers/politique-dutilisation/ 


\section{Intervenir en contexte(s) minoritaire(s)}

\section{Michèle Kérisit et Gertrude Mianda}

La diversité de la population ontarienne n'est plus à redire et elle est ancienne. Diversité originelle, tout d'abord, parmi ceux dont les ancêtres sont nés en sol ontarien, entre Cris,Anishnabe, Nations iroquoises et algonquines. Diversité, ensuite, entre les «autochtones» et les colons qui, eux-mêmes, se sont trouvés divisés entre Canadiens français et Loyalistes, entre francophones et anglophones originaires des Îles britanniques et d'Irlande. Puis au tournant de ce siècle, l'arrivée en grand nombre de nouveaux immigrants venus d'autres pays d'Europe (ils furent plus de 400000 à s'installer au Canada pour la seule année de 1913). Puis, depuis la fin des années 70, l'arrivée de nombreux immigrants venus d'Asie, d'Amérique du sud, des Caraibes, du Moyen Orient et d'Afrique. Entre 1991 et mai 1996, l'Ontario a accueilli 562985 nouveaux immigrants, soit plus de la moitié du nombre total d'immigrants s'installant au Canada entre ces deux dates (Statistique Canada 1997). Parmi ces nouveaux arrivants, quatre sur dix se sont établis à Toronto. Toutes les grandes villes de l'Ontario ont aussi connu un renouveau de l'immigration que ce soit Hamilton, Sudbury ou Ottawa.

Lorsque nous avons imaginé présenter aux lecteurs un numéro de Reflets portant sur l'intervention en contextes minoritaires, nous avions en tête non seulement la question de l'accueil des nouveaux arrivants francophones en Ontario, mais aussi celle de la diversité ontarienne dans son ensemble. Nous avons effectivement reçu des textes portant sur la situation et la complexité des pratiques d'intervention auprès de clientèles dont l'origine et la 
culture sont différentes des intervenantes et intervenants (personnes immigrantes et réfugiées et Premières Nations). Nous avons aussi reçu des articles décrivant certains défis de l'intervention en français en milieu majoritairement anglophone. Signalons cependant que nous n'avons reçu aucun texte traitant de la diversité au sein même de la population franco-ontarienne née en Ontario. Pourtant, les conditions de pratique dans le Nord sont sans doute différentes de celles du Sud ou de l'Est de l'Ontario. Peut-être faudra-t-il remettre à un autre numéro l'exploration de cette diversité régionale...

Par contre, nous devons signaler une innovation. Ce numéro de Reflets a en effet invité certains auteurs à nous tenir au courant des derniers développements de politiques sociales, quel que soit le thème central du numéro. Nous espérons ainsi que ces articles vous apporteront information et analyse des derniers changements dans deux domaines: la politique sur le travail obligatoire (Cholette) et la loi connue sous les termes de Loi sur la protection des locataires (Barnes). Selon Chantal Cholette, le programme de travail obligatoire «Ontario au travail» est un des éléments clés de la réforme du système de l'aide sociale en Ontario. Toutefois, plutôt que d'offrir de véritables solutions au chômage, ce programme met fin à d'importants acquis sociaux qui auront (et ont déjà, sans doute) des répercussions inévitables sur les intervenantes et les intervenants. La nouvelle loi sur la protection des locataires aura pour effet, selon Barnes, une augmentation des problèmes de logement. Il invite donc les intervenants et intervenantes concernés (ils sont nombreux) à se renseigner sur les changements contenus dans cette loi en donnant les coordonnées d'un certain nombre d'organismes qui suivent de près la question. Les bouleversements récents en Ontario et la restructuration des services affectent beaucoup les programmes et ressources en français selon Anne Simard, qui a conduit une étude à ce sujet pour le Centre ontarien d'information en prévention et le Centre for Health Promotion de l'Université de Toronto.

En publiant des textes portant en grande majorité sur l'intervention en contexte minoritaire, nous avons fait le pari qu'ils pourraient susciter une discussion approfondie sur des questions 
souvent laissées dans l'ombre car jugées très «délicates». Les textes publiés ici soulèvent, à notre avis, trois types de questions : en premier lieu, la question de l'identité franco-ontarienne que l'apport d'autres façons de faire et de voir le monde oblige peutêtre à réexaminer sous un autre angle; en deuxième lieu, la question de l'insertion spécifiquement minoritaire des francophones de l'Ontario et les effets de cette minorisation non seulement sur les pratiques quotidiennes des gens mais aussi sur les pratiques des intervenantes et des intervenants francophones; en troisième lieu, la question des contraintes qui pèsent sur la vie quotidienne des nouveaux arrivants et qu'une intervention interculturelle appropriée pourrait au moins soulager. Les auteurs de ce numéro ont évoqué, à leur façon, l'une de ces trois questions et ont tenté d'apporter des pistes de solution ou de réflexion afin d'aider les praticiens à relever le défi de la diversité.

L'identité peut être posée en termes linguistiques, culturels, mais surtout se forge au sein de relations entre personnes et entre groupes qui ont tendance à voir dans l'Autre, dans «celui-quin'est-pas comme-nous», la source du malaise provoqué par la rencontre. Plusieurs textes s'attachent à repenser cette rencontre entre personnes et groupes, qu'il soient composés de nouveaux arrivants, de Franco-Ontariens nés en Ontario ou qu'ils appartiennent au groupe majoritaire de langue anglaise.

Michèle Kérisit reprend la question des tensions qui président à l'insertion des nouveaux arrivants de langue française dans la communauté franco-ontarienne. Pour elle, ces défis naissent de deux logiques distinctes qui, cependant, se renforcent : d'une part une logique politique forgée dans l'histoire des relations entre majorité de langue anglaise et minorité de langue française au Canada, logique sur laquelle les nouveaux arrivants n'ont aucune prise; d'autre part, une logique proprement identitaire qui tend à faire se rapprocher les gens de même origine, à créer des «forteresses» difficilement accessibles aux nouveaux venus. Pourtant, selon l'auteure, nous sommes tous multiples et devrions utiliser la multiplicité de nos positionnements identitaires - en particulier notre expérience de minoritaires - afin de créer des ponts. Encore faut-il bien sûr reconnaitre qu'il existe des enjeux 
politiques posés par la hiérarchisation des rapports entre groupes minorisés. Une intervention interculturelle réussie ne peut donc passer que par «une prise de conscience de cette échelle de subordination, construite au nom de la culture mais en fait redevable de rapports sociaux et raciaux historiquement élaborés au sein même du Canada».

Ghislaine Sirois aborde également la question identitaire mais dans la perspective des relations entre majorité anglophone et minorité francophone. Grâce à des témoignages qu'elle a glanés au long des années, elle montre les effets de la minorisation du fait francophone sur les conditions de vie et de travail des intervenantes travaillant dans le domaine de la violence:travail multiplié et ardu, mais aussi absence d'écoute de la part du groupe majoritaire. En approfondissant également les dimensions psychologiques de la minorisation, l'auteure évoque la nécessité de se montrer peut-être plus active et actif dans la dénonciation de son «oppression». L'identité n'est pas, en effet, une donnée fixée une fois pour toutes mais se construit en relation à l'autre ou aux autres, et parfois en opposition. Son texte appelle certainement à discussion; elle l'a d'ailleurs écrit dans ce but. Nous espérons que son objectif sera entendu.

La question de l'identité est également au centre de l'article de Natalie Beausoleil qui s'est posé la question de savoir comment les Franco-Ontariennes qu'elle a interviewées définissaient leur identité à travers leur rapport au corps. Peut-on trouver dans leurs propos sur la santé, le vieillissement, le poids ou l'apparence, des signes qui les distinguereaient de la majorité anglo-saxonne de la province? Dans quelle mesure le fait d'être femme et d'être donc au confluent de normes souvent contradictoires quant à son corps s'articule-t-il à l'identité ethnique? Bien que prudente dans son interprétation, l'auteure esquisse une réflexion sur cette articulation entre ethnicisation et féminisation du corps en Ontario français. Elle n'en conclut pas moins que les femmes qu'elle a rencontrées possèdent une lucidité critique vis à vis des normes de féminité qu'il faudrait prendre en compte dans l'intervention. 
Bien qu'abordant la question sous un angle différent, Lamine Diallo et Ginette Lafrenière nous invitent aussi à comprendre certains enjeux liés à la question identitaire. En relatant la façon dont s'est créé le Groupe interculturel francophone du Moulin à fleur de Sudbury, ils nous montrent comment un travail de fond a pu faire se rencontrer un groupe de Franco-Ontariens, de nouveaux arrivants originaires d'Afrique mais aussi de francophones venant d'autres provinces, malgré des objectifs différents. Cependant, comme le soulignent les auteurs, les solidarités sont difficiles à créer. Seules la concertation et la reconnaissance des apports réciproques de chaque communauté peuvent surmonter les obstacles. La réflexion sur les relations interculturelles doit donc se penser en termes de représentativité au sein même de la communauté de langue française en Ontario.

Plusieurs autres auteurs de ce numéro illustrent comment cette représentativité au sein même des organismes peut se vivre et ce qu'elle peut apporter. Lisa Weintraub nous invite à comprendre comment l'organisme dont elle fait partie a, malgré certaines réticences de départ, cherché à rendre son personnel représentatif de la communauté francophone de HamiltonWentworth / Niagara. En transformant l'environnement physique, en mettant l'accent sur les rencontres informelles, en appliquant une politique d'embauche attentive aux questions de racisme et de rencontre interculturelle, le centre s'est petit à petit transformé pour répondre aux besoins de tous.

Elle n'est pas la seule à souligner la difficulté d'une telle entreprise, thème qui revient souvent sous la plume de nos auteurs. Mathilde Nduwimana nous présente son expérience d'intervenante interculturelle et nous montre combien il est difficile de travailler «à cheval» entre deux mondes. Tiraillés entre des valeurs et des besoins immédiats qu'ils connaissent et les mandats des organismes pour lesquels ils travaillent, les intervenantes et les intervenants se retrouvent souvent dans une position peu enviable. De plus, la structure même des "projets multiculturels», souvent subventionnés pour très peu de temps, fait qu'il leur est difficile de mesurer l'impact de leurs actions. Une refonte de la façon dont sont conçus les projets, un soutien mutuel 
entre intervenants rencontrant les même défis sembleraient des solutions possibles.

La difficulté du travail «interculturel» fait aussi l'objet d'une réflexion de la part de Richard Monette. Engagé dans un projet de développement communautaire avec des familles somaliennes dans la Basse-Ville d'Ottawa, l'auteur décrit comment les processus d'élaboration de projets comnmunautaires, qui doivent impliquer les gens concernés, correspondent peu aux attentes beaucoup plus immédiates d'une population particulièrement démunie. Alors que les activités directement accessibles connaissent un certain succès, les aleas d'un projet communautaire, dont on ne voit pas le résultat immédiat et qui ne bénéficiera que d'un financement ponctuel, ne suscitent pas une participation marquée. La question qui se pose alors est de trouver des partenaires dans une communauté élargie et de miser sur les mesures de soutien aux enfants. La flexibilité, le temps et la prise en compte des préoccupations de base des clientèles semblent donc être les clés du succès.

Les défis du développement communautaire dans une communauté divisée par la langue et la culture sont aussi évoqués par Nancy Blanchette. Un projet de développement des habiletés d'emploi de jeunes autochtones dans la région de Hearst a connu un départ difficile en raison de conflits dépassant le projet luimême. L'objectif de sensibilisation aux valeurs autochtones n'a pas pu se réaliser. Par contre, en se centrant sur la création d'une entreprise de formation en milieu de travail, l'organisme a réussi à concentrer les énergies des participantes et des participants, malgré les contraintes budgétaires qui empêchent de rendre le service disponible à tous. Ici encore, la flexibilité et la prise en considération des besoins immédiats ont réussi à faire en sorte qu'un dialogue s'instaure au-delà des préjugés et des conflits.

Est-ce à dire, pour autant, que l'intervention interculturelle ne comporte que des défis? Certes non. Plusieurs articles dans ce numéro de Reflets examinent comment l'on pourrait apprendre de pratiques développées différemment. Le texte d'Adje van de Sande et de Gilles Renault nous donne certaines clés au développement de pratiques centrées sur des principes autochtones, en 
particulier la roue médicinale. Confiants dans le succès de ce type d'intervention, les auteurs mettent en avant la nécessité de baser l'intervention sur une reconnaisssance du sens que les participants aux Cercles de partage donnent aux événements souvent très bouleversants de leur vie. Le témoignage des auteurs est d'ailleurs ancré dans leur volonté d'introduire cette façon de faire dans la formation des intervenantes et des intervenants, préoccupation pédagogique qui traverse d'autres textes du recueil.

Le témoignage de Claire Parrot s'ouvre également sur l'enrichissement que peut apporter une rencontre avec des personnes venant d'horizons différents de celui des intervenants et des intervenantes. Basant son texte sur son expérience d'infirmièreconseil en santé sexuelle et admettant les distances qui séparent les perspectives nord-américaines des perspectives africaines, en particulier, l'auteure met en lumière l'apprentissage qu'apportent le contact et la rencontre de ces perspectives. Il n' en reste pas moins que cet apprentissage reste lié à la possibilité de se départir des préjugés les plus communs sur ceux qu'on pense "différents». Elle aussi, comme d'autres auteurs dans ce numéro, favorise la reconnaissnce des multiples façons qui existent d'être «humain» et des multiples identités que nous portons en nous. La question des préjugés et des perceptions fait l'objet du texte de Myrtha Lapierre-Peters. Elle s'est demandé quelles perceptions les personnes âgées blanches et franco-ontariennes avaient des soignantes de «minorité visible», le plus souvent originaires d'Haïti. Elle rapporte ainsi les propos de ces personnes peu habituées à la rencontre de francophones nées sous d'autres cieux, qu'ils soient négatifs ou positifs. Elle explore également les perceptions des soignantes elles-mêmes. Ce qui frappe alors, c'est la volonté des intervenantes de poursuivre un dialogue qu'elles considèrent comme essentiel à leur professionnalisme, malgré certaines attitudes négatives.

Comme vous l'aurez noté, la majorité des articles publiés dans ce numéro de Reflets porte sur les rapports entre Ontariens francophones venus de plusieurs coins du monde et Ontariens francphones nés ici. Chacun, à sa façon, reconnaît l'importance de prendre en considération les cultures et les façons de faire des 
personnes auprès de qui les intervenants travaillent. Aucun, cependant, ne nous propose de recettes toutes faites, et en tant que directrices de ce numéro, nous sommes tout à fait en accord avec une telle approche. La question en effet n'en est pas tant une de «techniques» d'intervention et de "savoir» sur telle ou telle culture que d'écoute extrêment active et de sensibilité aux histoires que les nouveaux arrivants ont à nous dire. Ceux-ci ont souvent traversé des épreuves que bien peu d'Ontariens nés ici peuvent s'imaginer. Dans cette mesure, ce numéro de Reflets prend naissance dans les bouleversements mondiaux qui ont lieu en Afrique, en Asie, ou dans les Antilles et se fait l'écho des multiples situations dans lesquelles peuvent se retrouver ces nouveaux arrivants. Chaque article reprend également à sa façon les difficultés que vivent les nouveaux arrivants : langue, chômage ou sous-emploi, accès aux services mais aussi chocs culturels. Ces difficultés sont d'ailleurs vécues différemment par les nouveaux arrivants. L'homogénéité nexiste pas plus parmi les nouveaux arrivants que parmi les francophones nés au Canada...

Les situations des immigrants sont multiples, en effet, et traversées par d'autres facteurs que l'immigration elle-même: on vit l'arrivée en Ontario différemment selon qu'on est homme ou femme, jeune ou adulte, riche ou démuni de moyens matériels. Dans Le dossier, Gertrude Mianda nous rapporte la façon dont des femmes immigrantes originaires de l'Afrique subsaharienne francophone vivent leurs difficultés d'intégration à Toronto et perçoivent les rapports entre hommes et femmes. Étant donné l'importance que les rapports familiaux ont dans la problématique de l'intégration et la fréquence des questions que se posent les intervenantes et les intervenants dans ce domaine, cet article nous permet de comprendre que ces rapports entre hommes et femmes sont souvent vécus dans la tension, en raison des contraintes socioéconomiques vécues au sein de la famille et en fonction de valeurs dont il est difficile de se départir, surtout quand elles constituent le dernier bastion du respect que l'on se doit à soi-même. Il est donc regrettable que cette tension ne soit perçue que sous l'angle de la "soumission» des femmes africaines par de nombreuses femmes blanches nées au Canada. Cette incompréhension, 
accentuée par les discriminations racistes et sexistes dont sont victimes les femmes africaines dans la société en général, renforce en fait des tensions qu'il s'agirait peut-être de dissoudre davantage. Dans le même ordre d'idée, Marie-Claude Muamba évoque les difficultés rencontrées non seulement au niveau de l'emploi mais aussi lors de la recherche de services de qualité et en français. Elle nous raconte la désillusion et les péripéties de l'immigrant ou de l'immigrante francophone en butte aux difficultés d'acccès à ces services. Elle en a conclu, rapidement, que le bilinguisme tant prôné au Canada (en particulier dans les missions étrangères) n'était, en fait qu'un «bilinguisme du dimanche». «Ma conclusion est la suivante», dit-elle, «anglais excellent, French so so, vous êtes bilingue»... En cela, elle rejoint bien d'autres francophones de l'Ontario, nés ici.

Préjugés, racisme, oppression et minorisation sont des termes souvent utilisés dans ce numéro de Reflets. Il n'est plus temps de se boucher les oreilles et de se couvrir les yeux afin d'ignorer que la francophonie ontarienne est en train de changer et que ces changements ont des répercussions importantes sur la façon dont nous devons penser les pratiques d'intervention. En effet, si nous devons donner une impulsion aux demandes de reconnaissance du fait français en Ontario, si nous sommes appelés à développer des services de qualité en français, il nous faudra, à toutes et à tous, accepter que la langue française se vit et s'épelle de bien des façons. Il nous faudra enfin reconnaitre que l'homogéinéité des communautés franco-ontariennes est à repenser, non seulement en termes de revenus et de classes sociales, non seulement en termes de relations sociales de sexe, comme le rappelaient les deux derniers numéros de Reflets, mais aussi en termes d'ethnicité. Nous souhaitons donc, en tant que directrices de ce numéro, que toutes ces composantes soient intégrées à la réflexion que font les travailleuses et travailleurs sociaux quand il est temps d'intervenir. 